Article

\title{
Conformally Flat Siklos Metrics Are Ricci Solitons
}

\author{
Giovanni Calvaruso \\ Dipartimento di Matematica e Fisica “E. De Giorgi”, Università del Salento, Prov. Lecce-Arnesano, \\ 73100 Lecce, Italy; giovanni.calvaruso@unisalento.it
}

Received: 17 May 2020; Accepted: 4 June 2020; Published: 8 June 2020

\begin{abstract}
We study and solve the Ricci soliton equation for an arbitrary locally conformally flat Siklos
\end{abstract} metric, proving that such spacetimes are always Ricci solitons.

Keywords: exact solutions; Siklos spacetimes; Ricci solitons; conformally flat metrics; gradient Ricci solitons

\section{Introduction}

A Ricci soliton is a triple $(M, g, X)$, where $(M, g)$ is a pseudo-Riemannian manifold and $X$ a smooth tangent vector field on $M$, such that

$$
\mathcal{L}_{X} g+\varrho=\lambda g,
$$

with $\mathcal{L}_{X}, \varrho$ and $\lambda$ respectively denoting the Lie derivative in the direction of $X$, the Ricci tensor and a real constant. The Ricci soliton is respectively called shrinking, steady or expanding, depending on whether $\lambda>0, \lambda=0$ or $\lambda<0$. Trivial solutions of the Ricci soliton Equation (1) are given by an Einstein manifold $(M, g)$ together with a Killing vector field $X$.

Ricci solitons have been originally introduced in the Riemannian case [1,2] and then investigated in pseudo-Riemannian settings. Several studies have been made in the Lorentzian case, with particular regard to spacetimes, because of their relevance in Theoretical Physics. Some examples of these studies may be found in [3-16] and references therein.

In a given system of local coordinates, the Ricci soliton equation is equivalent to an overdetermined system of nonlinear second order PDEs, whose resolution is in general very hard, and often hopeless.

When trying to solve completely the Ricci soliton equation, it is natural to consider manifolds admitting some Killing vector fields. In fact, the solutions of the Ricci soliton Equation (1) are determined up to a Killing vector field: if $Y$ is a Killing vector field and $X$ satisfies (1), then also $X+Y$ satisfies the Ricci soliton equation. This fact can make somehow easier to determine solutions of the Ricci soliton equation. Indeed, several examples of complete classifications of Ricci solitons have been obtained for Lorentzian manifolds which, being homogeneous (at least, spatially), admit a large Lie algebra of killing vector fields (see for example $[4,8,9,11,14]$ ).

The purpose of this paper is to study and solve the Ricci soliton equation for conformally flat Siklos metrics, that is, for Siklos metrics which are in the conformal class of a flat metric. Siklos metrics [17] are a class of spacetimes, solving Einstein's field equations with an Einstein-Maxwell source. They are of Petrov type $N$ with cosmological constant $\Lambda<0$ and always admit a null non-twisting Killing field. The whole class of Siklos metrics, in global coordinates $(v, u, x, y)=\left(x_{1}, x_{2}, x_{3}, x_{4}\right)$, is given by

$$
g=-\frac{3}{\Lambda x_{3}^{2}}\left(2 d x_{1} d x_{2}+H d x_{2}^{2}+d x_{3}^{2}+d x_{4}^{2}\right)
$$

where $H=H\left(x_{2}, x_{3}, x_{4}\right)$ is an arbitrary smooth function (see $\left.[17,18]\right)$. Such metrics have been studied under several different points of view. As proved in [18], they are exact gravitational waves propagating 
in the anti-de Sitter universe. Moreover, they coincide with the Kundt spacetimes belonging to the subclass $(I V)_{0}[19]$.

In [11], the author investigated the Ricci soliton equation for the one-parameter class of homogeneous Siklos spacetimes determined by $H= \pm x_{3}^{2 k}$, where $k$ is a real constant. We shall now make a corresponding study for the large class of locally conformally flat Siklos metrics, which depend on four arbitrary functions of one variable. After describing explicitly these metrics, we completely solve the Ricci soliton equation, proving the following main result.

Theorem 1. All non-Einstein conformally flat Siklos metrics are expanding Ricci solitons. These Ricci solitons are not of gradient type.

We recall that a gradient Ricci soliton satisfies Equation (1) for some vector field $X=\operatorname{grad}_{g}(f)$, where $f$ is a smooth function.

The paper is organized in the following way. In Section 2 we shall report some needed information on the curvature of Siklos metrics and determine explicitly the general form of the locally conformally flat examples. In Section 3 we shall prove that all these metrics are Ricci solitons, also proving that the solutions are never of gradient type. Completely explicit solutions are obtained in Section 4 for all conformally flat Siklos metrics admitting some additional Killing vector fields. Calculations have been checked using the software Maple 16 (C).

\section{Locally Conformally Flat Siklos Spacetimes}

We may refer to $[11,18]$ for the description of the Levi-Civita connection and curvature of an arbitrary Siklos metric $g$. We shall report below the information we need to write down the Ricci soliton equation and to identify the locally conformally flat cases.

With respect to the system of global coordinates $\left(x_{1}, x_{2}, x_{3}, x_{4}\right)$ used in (2), the Levi-Civita connection $\nabla$ of $g$ is completely determined by the following possibly non-vanishing components:

$$
\begin{array}{ll}
\nabla_{\partial_{1}} \partial_{2}=\frac{1}{x_{3}} \partial_{3}, & \nabla_{\partial_{1}} \partial_{3}=-\frac{1}{x_{3}} \partial_{1}, \\
\nabla_{\partial_{2}} \partial_{2}=\frac{1}{2}\left(\partial_{2} H\right) \partial_{1}+\frac{1}{2 x_{3}}\left(2 H-x_{3} \partial_{3} H\right) \partial_{3}-\frac{1}{2}\left(\partial_{4} H\right) \partial_{4}, & \nabla_{\partial_{2}} \partial_{3}=\frac{1}{2}\left(\partial_{3} H\right) \partial_{1}-\frac{1}{x_{3}} \partial_{2}, \\
\nabla_{\partial_{2}} \partial_{4}=\frac{1}{2}\left(\partial_{4} H\right) \partial_{1}, & \nabla_{\partial_{3}} \partial_{3}=-\frac{1}{x_{3}} \partial_{3}, \\
\nabla_{\partial_{3}} \partial_{4}=-\frac{1}{x_{3}} \partial_{4}, & \nabla_{\partial_{4}} \partial_{4}=\frac{1}{x_{3}} \partial_{3} .
\end{array}
$$

In particular, as proved in [11], Siklos metrics do not admit any parallel vector field. Consequently, they are neither locally reducible, nor strictly Walker manifolds (i.e., Lorentzian manifolds admitting a parallel null vector field).

The possibly non-vanishing components of the Riemann-Christoffel curvature tensor $R$ of $g$ are the following:

$$
\begin{array}{lll}
R_{1212}=-\frac{3}{\Lambda x_{3}^{4}}, & R_{1323}=\frac{3}{\Lambda x_{3}^{4}}, & R_{1424}=\frac{3}{\Lambda x_{3}^{4}}, \\
R_{2323}=\frac{3\left(2 H-x_{3}\left(\partial_{3} H\right)+x_{3}^{2}\left(\partial_{33}^{2} H\right)\right)}{2 \Lambda x_{3}^{4}}, & R_{2324}=\frac{3\left(\partial_{34}^{2} H\right)}{2 \Lambda x_{3}^{2}}, & R_{2424}=\frac{3\left(2 H-x_{3}\left(\partial_{3} H\right)+x_{3}^{2}\left(\partial_{44}^{2} H\right)\right)}{2 \Lambda x_{3}^{4}}, \\
R_{3434}=-\frac{3}{\Lambda x_{3}^{4}} & &
\end{array}
$$


and the Ricci tensor of $g$, defined by $\varrho(X, Y)=\operatorname{tr}(Z \mapsto R(Z, X) Y)$, is completely described by the matrix

$$
\varrho=\left(\begin{array}{cccc}
0 & -3 x_{3}^{-2} & 0 & 0 \\
-3 x_{3}^{-2} & -\frac{6 H-2 x_{3}\left(\partial_{3} H\right)+x_{3}^{2}\left(\partial_{33}^{2} H+\partial_{44}^{2} H\right)}{2 x_{3}^{2}} & 0 & 0 \\
0 & 0 & -3 x_{3}^{-2} & 0 \\
0 & 0 & 0 & -3 x_{3}^{-2}
\end{array}\right)
$$

in terms of its components with respect to $\left\{\partial_{i}\right\}$. Consequently, the Ricci operator Ric, defined by condition $\varrho(X, Y)=g(\operatorname{Ric}(X), Y)$, is described with respect to $\left\{\partial_{i}\right\}$ by the matrix

$$
\text { Ric }=\left(\begin{array}{cccc}
\Lambda & -\frac{1}{6} \Lambda x_{3}\left\{2\left(\partial_{3} H\right)-x_{3}\left(\partial_{33}^{2} H+\partial_{44}^{2} H\right)\right\} & 0 & 0 \\
0 & \Lambda & 0 & 0 \\
0 & 0 & \Lambda & 0 \\
0 & 0 & 0 & \Lambda
\end{array}\right) .
$$

In particular, the scalar curvature of a Siklos metric is then given by $\tau=4 \Lambda$. Moreover, the following result holds (see $[11,17,18]$ ).

Proposition 1. For an arbitrary Siklos metric $g$, as described in (2), the following conditions are equivalent:

(i) $g$ is Einstein. More precisely, $\varrho=\Lambda g$;

(ii) $g$ is Ricci-parallel (that is, $\nabla \varrho=0$ );

(iii) the defining function $H=H\left(x_{2}, x_{3}, x_{4}\right)$ satisfies the PDE

$$
\frac{2}{x_{3}}\left(\partial_{3} H\right)-\partial_{33}^{2} H-\partial_{44}^{2} H=0
$$

Whenever H does not satisfy (7), a Siklos spacetime (being not Ricci-parallel) is not locally symmetric, and its Ricci operator is of Segre type $(11,2)$, having an eigenvalue of multiplicity four associated to a three-dimensional eigenspace.

Next, we briefly recall that a pseudo-Riemannian manifold $(M, g)$ is said to be (locally) conformally flat when there exits (at least, locally) some smooth function $\phi$, such that $g=e^{\phi} \cdot g_{0}$, where $g_{0}$ is a flat metric. Locally conformally flat metrics are an important topic both in Riemannian and pseudo-Riemannian (in particular, Lorentzian) geometry. It is well known that in dimension greater than three, a pseudo-Riemannian manifold $(M, g)$ is conformally flat if and only if its Weyl conformal curvature tensor $W$ vanishes. The Weyl conformal curvature tensor field $C$ of $(M, g)$ is defined by

$$
C(X, Y) Z=R(X, Y) Z-\frac{1}{n-2}(Q X \wedge Y+X \wedge Q Y) Z+\frac{S}{(n-1)(n-2)}(X \wedge Y) Z,
$$

where $R, Q$ and $S$ respectively denote the curvature tensor, the Ricci operator and the scalar curvature, and $(X \wedge Y)(Z)=\langle Y, Z\rangle X-\langle X, Z\rangle Y$. It is well known that because of Equation (8), if $C=0$ then the Ricci curvature completely determines the curvature of $(M, g)$.

By (2), (4) and (5), a standard computation shows that for an arbitrary Siklos metric, the Weyl conformal curvature tensor $W$ is completely determined by the following possibly non-vanishing components $W_{i j k h}$ with respect to $\left\{\partial_{i}\right\}$ :

$$
W_{2323}=-W_{2424}=\frac{3}{4 \Lambda x_{3}^{2}}\left(\partial_{33}^{2} H-\partial_{44}^{2} H\right), \quad W_{2324}=\frac{3}{2 \Lambda x_{3}^{2}} \partial_{34}^{2} H .
$$


Thus, integrating the system of PDEs

$$
\frac{3}{4 \Lambda x_{3}^{2}}\left(\partial_{33}^{2} H-\partial_{44}^{2} H\right)=\frac{3}{2 \Lambda x_{3}^{2}} \partial_{34}^{2} H=0,
$$

we obtain the following.

Proposition 2. A Siklos metric $g$, as described in (2), is locally conformally flat if and only if the defining function $H=H\left(x_{2}, x_{3}, x_{4}\right)$ satisfies the system of PDEs

$$
\left\{\begin{array}{l}
\partial_{33}^{2} H-\partial_{44}^{2} H=0, \\
\partial_{34}^{2} H=0,
\end{array}\right.
$$

that is, when $H$ is explicitly given by

$$
H\left(x_{2}, x_{3}, x_{4}\right)=\frac{1}{2} T\left(x_{2}\right)\left(x_{3}^{2}+x_{4}^{2}\right)+L\left(x_{2}\right) x_{3}+M\left(x_{2}\right) x_{4}+N\left(x_{2}\right),
$$

where $T, L, M, N$ are arbitrary smooth functions.

It is easy to check that an arbitray conformally flat Siklos metric, as described by Equation (2) with $H$ given by (11), is Einstein (that is, satisfies condition (7)) if and only if $L\left(x_{2}\right)=0$. In this case $H=T\left(x_{2}\right)\left(x_{3}^{2}+x_{4}^{2}\right)+M\left(x_{2}\right) x_{4}+N\left(x_{2}\right)$ and one gets the Anti-De Sitter space [17].

\section{The Ricci Soliton Equation for Conformally Flat Siklos Metrics}

With respect to the system of global coordinates $\left(x_{1}, x_{2}, x_{3}, x_{4}\right)$ used to describe Siklos metrics in (2), consider an arbitrary vector field $X=X_{i} \partial_{i}$, where $X_{i}=X_{i}\left(x_{1}, x_{2}, x_{3}, x_{4}\right), i=1, \ldots, 4$, are smooth functions. The Lie derivative $\mathcal{L}_{X} g$ is completely determined by the components $\left(\mathcal{L}_{X} g\right)_{i j}=\left(\mathcal{L}_{X} g\right)\left(\partial_{i}, \partial_{j}\right), i \leq j=1, \ldots, 4$, and can be obtained by direct computation using (3). Explicitly, we get:

$$
\left\{\begin{array}{l}
\left(\mathcal{L}_{X} g\right)_{11}=-\frac{6}{\Lambda x_{3}^{2}} \partial_{1} X_{2}, \\
\left(\mathcal{L}_{X} g\right)_{12}=-\frac{3}{\Lambda x_{3}^{3}}\left\{x_{3} \partial_{1} X_{1}+x_{3} H \partial_{1} X_{2}+x_{3} \partial_{2} X_{2}-2 X_{3}\right\}, \\
\left(\mathcal{L}_{X} g\right)_{13}=-\frac{3}{\Lambda x_{3}^{2}}\left\{\partial_{3} X_{2}+\partial_{1} X_{3}\right\}, \\
\left(\mathcal{L}_{X} g\right)_{14}=-\frac{3}{\Lambda x_{3}^{2}}\left\{\partial_{4} X_{2}+\partial_{1} X_{4}\right\}, \\
\left(\mathcal{L}_{X} g\right)_{22}=-\frac{3}{\Lambda x_{3}^{3}}\left\{2 x_{3} \partial_{2} X_{1}+x_{3} \partial_{2} H X_{2}+2 x_{3} H \partial_{2} X_{2}-2 H X_{3}+x_{3} \partial_{3} H X_{3}+x_{3} \partial_{4} H X_{4}\right\}, \\
\left(\mathcal{L}_{X} g\right)_{23}=-\frac{3}{\Lambda x_{3}^{2}}\left\{\partial_{3} X_{1}+H \partial_{3} X_{2}+\partial_{2} X_{3}\right\}, \\
\left(\mathcal{L}_{X} g\right)_{24}=-\frac{3}{\Lambda x_{3}^{2}}\left\{\partial_{4} X_{1}+H \partial_{4} X_{2}+\partial_{2} X_{4}\right\}, \\
\left(\mathcal{L}_{X} g\right)_{33}=-\frac{6}{\Lambda x_{3}^{3}}\left\{x_{3} \partial_{3} X_{3}-X_{3}\right\}, \\
\left(\mathcal{L}_{X} g\right)_{34}=-\frac{3}{\Lambda x_{3}^{2}}\left\{\partial_{4} X_{3}+\partial_{3} X_{4}\right\}, \\
\left(\mathcal{L}_{X} g\right)_{44}=-\frac{6}{\Lambda x_{3}^{3}}\left\{x_{3} \partial_{4} X_{4}-X_{3}\right\} .
\end{array}\right.
$$

Using the above components of $\mathcal{L}_{X} g$, together with the ones of the metric tensor $g$ and the Ricci tensor $\varrho$ with respect to the basis $\left\{\partial_{i}\right\}$, we find that an arbitrary Siklos metric $g$, together with the 
vector field $X$, satisfies the Ricci soliton Equation (1) if and only if the components $X_{i}$ of vector field $X$ satisfy the following system of ten PDEs:

$$
\left\{\begin{array}{l}
\partial_{1} X_{2}=0 \\
x_{3} \partial_{1} X_{1}+x_{3} H \partial_{1} X_{2}+x_{3} \partial_{2} X_{2}-2 X_{3}+(\Lambda-\lambda) x_{3}=0 \\
\partial_{3} X_{2}+\partial_{1} X_{3}=0 \\
\partial_{4} X_{2}+\partial_{1} X_{4}=0 \\
12 x_{3} \partial_{2} X_{1}+6 x_{3} \partial_{2} H X_{2}+12 x_{3} H \partial_{2} X_{2}-12 H X_{3}+6 x_{3} \partial_{3} H X_{3}+6 x_{3} \partial_{4} H X_{4} \\
-2 \Lambda x_{3}^{2} \partial_{3} H+\Lambda x_{3}^{3}\left(\partial_{33}^{2} H+\partial_{44}^{2} H\right)+6(\Lambda-\lambda) x_{3} H=0 \\
\partial_{3} X_{1}+H \partial_{3} X_{2}+\partial_{2} X_{3}=0 \\
\partial_{4} X_{1}+H \partial_{4} X_{2}+\partial_{2} X_{4}=0 \\
2 x_{3} \partial_{3} X_{3}-2 X_{3}+(\Lambda-\lambda) x_{3}=0 \\
\partial_{4} X_{3}+\partial_{3} X_{4}=0 \\
2 x_{3} \partial_{4} X_{4}-2 X_{3}+(\Lambda-\lambda) x_{3}=0
\end{array}\right.
$$

We start integrating the first equation in (13), obtaining

$$
X_{2}=F_{2}\left(x_{2}, x_{3}, x_{4}\right),
$$

for some smooth function $F_{2}$. Substituting from (14) into the third and fourth equations of (13) and integrating, we respectively get

$$
\left\{\begin{array}{l}
X_{3}=-x_{1} \partial_{3} F_{2}\left(x_{2}, x_{3}, x_{4}\right)+F_{3}\left(x_{2}, x_{3}, x_{4}\right), \\
X_{4}=-x_{1} \partial_{4} F_{2}\left(x_{2}, x_{3}, x_{4}\right)+F_{4}\left(x_{2}, x_{3}, x_{4}\right),
\end{array}\right.
$$

where $F_{3}, F_{4}$ are smooth functions. Because of (15), the ninth equation of (13) becomes

$$
2 \partial_{34}^{2} F_{2}\left(x_{2}, x_{3}, x_{4}\right) x_{1}-\left(\partial_{4} F_{3}\left(x_{2}, x_{3}, x_{4}\right)+\partial_{3} F_{4}\left(x_{2}, x_{3}, x_{4}\right)\right)=0,
$$

which, as a polynomial equation in the variable $x_{1}$, implies at once

$$
\partial_{34}^{2} F_{2}\left(x_{2}, x_{3}, x_{4}\right)=\partial_{4} F_{3}\left(x_{2}, x_{3}, x_{4}\right)+\partial_{3} F_{4}\left(x_{2}, x_{3}, x_{4}\right)=0 .
$$

In particular, integrating $\partial_{34}^{2} F_{2}\left(x_{2}, x_{3}, x_{4}\right)=0$, we get

$$
F_{2}\left(x_{2}, x_{3}, x_{4}\right)=G_{2}\left(x_{2}, x_{3}\right)+H_{2}\left(x_{2}, x_{4}\right),
$$

for some smooth functions $G_{2}, H_{2}$. We apply the same argument and use the above expressions into the eighth equation of (13), which becomes a polynomial in the variable $x_{1}$. Setting equal to zero the coefficient of $x_{1}$, we get

$$
x_{3} \partial_{33}^{2} G_{2}\left(x_{2}, x_{3}\right)-\partial_{3} G_{2}\left(x_{2}, x_{3}\right)=0
$$

whence, by integration,

$$
G_{2}\left(x_{2}, x_{3}\right)=A_{2}\left(x_{2}\right)+B_{2}\left(x_{2}\right) x_{3}^{2},
$$

for some smooth functions $A_{2}, B_{2}$. The eighth equation of (13) then reduces to

$$
2 x_{3} \partial_{3} F_{3}\left(x_{2}, x_{3}, x_{4}\right)-2 F_{3}\left(x_{2}, x_{3}, x_{4}\right)+(\Lambda-\lambda) x_{3}=0,
$$


which by integration gives

$$
F_{3}\left(x_{2}, x_{3}, x_{4}\right)=\left(\frac{\lambda-\Lambda}{2} \ln \left(x_{3}\right)+G_{3}\left(x_{2}, x_{4}\right)\right) x_{3},
$$

for some smooth function $G_{3}$. Next, applying the above expressions, the ninth equation in (13) becomes

$$
x_{3} \partial_{4} G_{3}\left(x_{2}, x_{4}\right)+\partial_{3} F_{4}\left(x_{2}, x_{3}, x_{4}\right)=0,
$$

whence

$$
F_{4}\left(x_{2}, x_{3}, x_{4}\right)=-\frac{1}{2} x_{3}^{2} \partial_{4} G_{3}\left(x_{2}, x_{4}\right)+G_{4}\left(x_{2}, x_{4}\right),
$$

for a smooth function $G_{4}$. The sixth equation in (13) now reduces to

$$
-\partial_{3} X_{1}-2 H B_{2}\left(x_{2}\right)+2 x_{1} x_{3} B_{2}^{\prime}\left(x_{2}\right)-x_{3} \partial_{2} G_{3}\left(x_{2}, x_{4}\right)=0
$$

and integrating we get

$$
X_{1}=\frac{1}{2}\left(2 x_{1} B_{2}^{\prime}\left(x_{2}\right)-\partial_{2} G_{3}\left(x_{2}, x_{4}\right)\right) x_{3}^{2}-2 B_{2}\left(x_{2}\right) \int\left(x_{3} H\right) d x_{3}+F_{1}\left(x_{1}, x_{2}, x_{4}\right),
$$

for a smooth function $F_{1}$. We then substitute the above expressions into the last equation of (13) and write it as a polynomial in $x_{1}$, obtaining

$$
\begin{aligned}
& 2\left(2 B_{2}\left(x_{2}\right)-\partial_{44}^{2} H_{2}\left(x_{2}, x_{4}\right)\right) x_{1} \\
& -\left(2 G_{3}\left(x_{2}, x_{4}\right)+x_{3}^{2} \partial_{44}^{2} G_{3}\left(x_{2}, x_{4}\right)-2 \partial_{4} G_{3}\left(x_{2}, x_{4}\right)+(\lambda-\Lambda)\left(\ln \left(x_{3}\right)+1\right)\right)=0 .
\end{aligned}
$$

Since the coefficients of such polynomial must necessarily vanish, by integration we find

$$
H_{2}\left(x_{2}, x_{4}\right)=x_{4}^{2} B_{2}\left(x_{2}\right)+x_{4} C_{2}\left(x_{2}\right)+D_{2}\left(x_{2}\right),
$$

with $C_{2}, D_{2}$ smooth functions, and (21) reduces to

$$
x_{3}^{2} \partial_{44}^{2} G_{3}\left(x_{2}, x_{4}\right)+(\lambda-\Lambda) \ln \left(x_{3}\right)+\left(2 G_{3}\left(x_{2}, x_{4}\right)-2 \partial_{4} G_{4}\left(x_{2}, x_{4}\right)+(\lambda-\Lambda)\right)=0,
$$

which is a linear combination of linearly independent functions $x_{3}^{2}, \ln \left(x_{3}\right)$ and $1=x_{3}^{0}$. Therefore, their coefficients must vanish, so that we get $\lambda=\Lambda$,

$$
\begin{aligned}
& G_{3}\left(x_{2}, x_{4}\right)=x_{4} A_{3}\left(x_{2}\right)+B_{3}\left(x_{2}\right), \\
& G_{4}\left(x_{2}, x_{4}\right)=\frac{1}{2} x_{4}^{2} A_{3}\left(x_{2}\right)+x_{4} B_{3}\left(x_{2}\right)+A_{4}\left(x_{2}\right),
\end{aligned}
$$

for a smooth function $A_{4}$. By the above equations, the second equation of (13) reduces to the following polynomial equation in $x_{3}$ :

$$
\begin{aligned}
& -2 x_{3}^{2} B_{2}^{\prime}\left(x_{2}\right)-\left\{\partial_{1} F_{1}\left(x_{1}, x_{2}, x_{4}\right)+A_{2}^{\prime}\left(x_{2}\right)+x_{4}^{2} B_{2}^{\prime}\left(x_{2}\right)+x_{4} C_{2}^{\prime}\left(x_{2}\right)+D_{2}^{\prime}\left(x_{2}\right)\right. \\
& \left.+4 x_{1} B_{2}\left(x_{2}\right)-2 x_{4} A_{3}\left(x_{2}\right)-2 B_{3}\left(x_{2}\right)\right\}=0
\end{aligned}
$$

which yields at once $B_{2}^{\prime}\left(x_{2}\right)=0$. Thus, $B_{2}\left(x_{2}\right)=b_{2}$ is a real constant, and the remaining part of (24) by integration gives

$$
F_{1}\left(x_{1}, x_{2}, x_{4}\right)=-2 b_{2} x_{1}^{2}-\left(A_{2}^{\prime}\left(x_{2}\right)+x_{4} C_{2}^{\prime}\left(x_{2}\right)+D_{2}^{\prime}\left(x_{2}\right)-2 x_{4} A_{3}\left(x_{2}\right)-2 B_{3}\left(x_{2}\right)\right) x_{1}+G_{1}\left(x_{2}, x_{4}\right),
$$


where $G_{1}$ is a smooth function. Next, we substitute from the above expressions into the seventh equation of (13). Writing it as a polynomial in the variable $x_{1}$, we find

$$
\begin{aligned}
& 4\left\{A_{3}\left(x_{2}\right)-C_{2}^{\prime}\left(x_{2}\right)\right\} x_{1}+\left\{2 \partial_{4} G_{1}\left(x_{2}, x_{4}\right)+\left(x_{4}^{2}-2 x_{3}^{2}\right) A_{3}^{\prime}\left(x_{2}\right)\right. \\
& \left.-4 b_{2} \int\left(x_{3} \partial_{4} H\right) d x_{3}+2\left(C_{2}\left(x_{2}\right)+2 b_{2} x_{4}\right) H+2 x_{4} B_{3}^{\prime}\left(x_{2}\right)+2 A_{4}^{\prime}\left(x_{2}\right)\right\}=0 .
\end{aligned}
$$

In particular, $A_{3}\left(x_{2}\right)=C_{2}^{\prime}\left(x_{2}\right)$.

Remark 1. Replacing from all the above expressions, the system (13) now reduces to its fifth and seventh equations. Observe that in so far we did not make any assumption on the defining function $H$, so that calculations above are valid for any metric (2) in the Siklos class.

In particular, whenever a Siklos metric satisfies the Ricci soliton equation, necessarily $\lambda=\Lambda$, so that the cosmological constant $\Lambda<0$ naturally appears in such equation, and the Ricci soliton is necessarily expanding.

In the remaining part of this paper we shall focus on the conformally flat cases. Thus, following the result of the previous section, we shall assume that the defining function $H$ of the Siklos metric is given by (11), depending on four arbitrary smooth functions $T, L, M, N$. Moreover, we shall assume $L\left(x_{2}\right) \neq 0$ everywhere, in order to exclude (even locally) the Einstein case.

We use the description (11) of $H$ into the two remaining equations of (13). The first of such equations now gives a first order polynomial function in the variable $x_{1}$, that is, one of the form $P\left(x_{2}, x_{3}, x_{4}\right) x_{1}+Q\left(x_{2}, x_{3}, x_{4}\right)=0$, so that necessarily $P=Q=0$. In particular, as

$$
\begin{aligned}
P\left(x_{2}, x_{3}, x_{4}\right)= & -12 b_{2} L\left(x_{2}\right) x_{3}+6\left\{\left(T\left(x_{2}\right) C_{2}\left(x_{2}\right)-2 b_{2} M\left(x_{2}\right)-2 C_{2}^{\prime \prime}\left(x_{2}\right)\right) x_{4}\right. \\
& \left.+M\left(x_{2}\right) C_{2}\left(x_{2}\right)-4 b_{2} N\left(x_{2}\right)-4 B_{3}^{\prime}\left(x_{2}\right)+2 A_{2}^{\prime \prime}\left(x_{2}\right)+2 D_{2}^{\prime \prime}\left(x_{2}\right)\right\}=0,
\end{aligned}
$$

which we have written down as a polynomial equation in $x_{3}$, taking into account $L\left(x_{2}\right) \neq 0$ we have $b_{2}=0$. Instead of integrating the remaining part of (26), we now replace from the above expressions into the seventh equation of (13) and write it as a second order polynomial in the variable $x_{3}$. The coefficient of $x_{3}$ in such polynomial equation yields $3 L\left(x_{2}\right) C_{2}\left(x_{2}\right)=0$, whence we get

$$
C_{2}\left(x_{2}\right)=0 .
$$

The seventh equation in (13) then reduces to

$$
\partial_{4} G_{1}\left(x_{2}, x_{4}\right)+x_{4} B_{3}^{\prime}\left(x_{2}\right)+A_{4}^{\prime}\left(x_{2}\right)=0,
$$

from which by integration we find

$$
G_{1}\left(x_{2}, x_{4}\right)=-\frac{1}{2} x_{4}^{2} B_{3}^{\prime}\left(x_{2}\right)-x_{4} A_{4}^{\prime}\left(x_{2}\right)+A_{1}\left(x_{2}\right),
$$

for some smooth function $A_{1}$. We are now left with the fifth equation of (13), which we write down as a first order polynomial equation in the variable $x_{1}$. From the coefficient of $x_{1}$ in this equation we find

$$
A_{2}^{\prime \prime}\left(x_{2}\right)+D_{2}^{\prime \prime}\left(x_{2}\right)-2 B_{3}^{\prime}\left(x_{2}\right)=0 .
$$

Thus, there exists some real constant $b_{3}$, such that

$$
B_{3}\left(x_{2}\right)=\frac{1}{2} A_{2}^{\prime}\left(x_{2}\right)+\frac{1}{2} D_{2}^{\prime}\left(x_{2}\right)+b_{3} .
$$

We set the integration function $D_{2}\left(x_{2}\right)=-A_{2}\left(x_{2}\right)$. By this expression and taking into account Equation (28), the fifth equation of (13), written now as a polynomial in the variable $x_{3}$, takes the form 
$P\left(x_{2}\right) x_{3}^{2}+Q\left(x_{2}, x_{4}\right) x_{3}=0$, whence $P=Q=0$. In particular, as $P\left(x_{2}\right)=-\left(\Lambda+3 b_{3}\right) L\left(x_{2}\right)$ and we assumed $L \neq 0$, we find

$$
b_{3}=-\frac{1}{3} \Lambda
$$

Thus, also using the above condition for $b_{3}$, we are left with the equation

$$
\begin{aligned}
Q\left(x_{2}, x_{4}\right)= & \left\{6 A_{4}^{\prime \prime}\left(x_{2}\right)-3 T\left(x_{2}\right) A_{4}\left(x_{2}\right)-\Lambda M\left(x_{2}\right)\right\} x_{4} \\
& -\left\{6 A_{1}^{\prime}\left(x_{2}\right)+3 M\left(x_{2}\right) A_{4}\left(x_{2}\right)+2 \Lambda N\left(x_{2}\right)\right\}=0,
\end{aligned}
$$

which we wrote down as a first degree polynomial equation in $x_{4}$, whose coefficients only depend on the variable $x_{2}$. By setting equal to zero such coefficients we determine $A_{1}\left(x_{2}\right)$ and $A_{4}\left(x_{2}\right)$ in function of terms $T\left(x_{2}\right), M\left(x_{2}\right), N\left(x_{2}\right)$ occurring in the defining function $H$. Explicitly, we get

$$
A_{1}\left(x_{2}\right)=-\int\left(\frac{1}{3} \Lambda N\left(x_{2}\right)+\frac{1}{2} M\left(x_{2}\right) A_{4}\left(x_{2}\right)\right) d x_{2}+C_{1}
$$

with $A_{4}$ completely determined as solution of

$$
A_{4}^{\prime \prime}\left(x_{2}\right)=\frac{1}{2} T\left(x_{2}\right) A_{4}\left(x_{2}\right)+\Lambda M\left(x_{2}\right) .
$$

Observe that the above Equation (30) is of type $A_{4}^{\prime \prime}(t)=f\left(t, A_{4}\right)$, with $f$ a smooth function of $t$ and $a_{4}$. Therefore, the standard existence theorem yields that a solution exists for all values of $t=x_{2}$.

Once $A_{4}$ is determined as a solution of (30), all equations of (13) are satisfied. The explicit description of the components $X_{i}$ of vector field $X$ with respect to $\left\{\partial_{i}\right\}$ are now obtained substituting all the previous formulas into (14), (15) and (20). We get that an arbitrary conformally flat Siklos metric (2), with $H$ determined as in (11), is an expanding Ricci soliton, for which Equation (1) holds with $\lambda=\Lambda<0$ and $X=X^{i} \partial_{i}$ of components

$$
\left\{\begin{array}{l}
X^{1}=-\frac{2}{3} \Lambda x_{1}-\int\left(\frac{1}{3} \Lambda N\left(x_{2}\right)+\frac{1}{2} M\left(x_{2}\right) A_{4}\left(x_{2}\right)\right) d x_{2}-A_{4}^{\prime}\left(x_{2}\right) x_{4}+C_{1} \\
X^{2}=0 \\
X^{3}=-\frac{1}{3} \Lambda x_{3} \\
X^{4}=A_{4}\left(x_{2}\right)-\frac{1}{3} \Lambda x_{4}
\end{array}\right.
$$

The above conclusion is confirmed by computing separately $\left(\mathcal{L}_{\mathrm{X}} g\right)_{i j}$ and $\Lambda g_{i j}-\varrho_{i j}$ for all indices $i, j=1, \ldots, 4$. In fact, by (2), (11), (5) and (31), taking into account (30) we find

$$
\begin{array}{ll}
\left(\mathcal{L}_{X} g\right)_{22}=-\frac{1}{x_{3}} L\left(x_{2}\right)=\Lambda g_{22}-\varrho_{22}, & \\
\left(\mathcal{L}_{X} g\right)_{i j}=0=\Lambda g_{i j}-\varrho_{i j} \quad \text { in the other cases, }
\end{array}
$$

so that Equation (1) holds with $\lambda=\Lambda$. It may be noticed that the Einstein case is characterized by condition $L\left(x_{2}\right)=0$, in which case the above equations hold trivially for a Killing vector field $X$ $\left(\mathcal{L}_{X} g=0\right)$ and $\varrho=\Lambda g$. Thus, we obtained the following result, which proves the first statement of Theorem 1 .

Theorem 2. Conformally flat Siklos metrics $g$, as described by (2) with the defining function $H$ given by (11), are expanding Ricci solitons, which satisfy Equation (1) with $\lambda=\Lambda$ for a vector field $X=X_{i} \partial_{i}$ described by (31), where $A_{4}$ is a smooth function satisfying (30). 
We end this section checking that conformally flat Siklos metrics are not gradient Ricci solitons. As already recalled in the Introduction, a Ricci soliton is said to be gradient when it satisfies Equation (1) for a vector field $X=\operatorname{grad}_{g}(f)$, where $f$ is a smooth function. In this case, Equation (1) becomes

$$
\operatorname{Hess}(f)+\varrho=\lambda g
$$

with Hess $(f)$ the Hessian of $f$. It is worthwhile to remark that the existence of a gradient Ricci soliton yields some strong restrictions in several classes of Lorentzian manifolds, and this is particularly true for the locally conformally flat ones. In fact, locally conformally flat Lorentzian gradient Ricci solitons have been completely described. As proved in [6], they are locally isometric

- to Robertson-Walker warped products of a real interval with a space of constant sectional curvature in the nonisotropic case $\left(\left\|\operatorname{grad}_{g}(f)\right\| \neq 0\right)$;

- $\quad$ to a plane wave in the isotropic case $\left(\left\|\operatorname{grad}_{g}(f)\right\|=0\right)$.

Locally conformally flat Siklos spacetimes do not fit in the above cases, but this does not yield to any contradiction, as they are Ricci solitons but not of gradient type. In fact, consider the solution of the Ricci soliton equation described in the above Theorem 2. If such Ricci soliton were gradient, then there would be some smooth function $f=f\left(x_{1}, x_{2}, x_{3}, x_{4}\right)$, such that vector $X=\operatorname{grad}_{g}(f)$.

We use (2) to determine the inverse matrix $g^{-1}=\left(g^{i j}\right)$ of the matrix describing the metric tensor $g$ in coordinates $\left(x_{1}, x_{2}, x_{3}, x_{4}\right)$ and then calculate $\operatorname{grad}_{g}(f)=\sum_{i, j} g^{i j} \frac{\partial f}{\partial x_{i}} \partial_{i}$. We find that $X=X_{i} \partial_{i}=$ $\operatorname{grad}_{g}(f)$, with $X_{i}$ described as in (31), if and only if the following system of 4 PDEs is satisfied:

$$
\left\{\begin{array}{l}
\Lambda x_{3}^{2}\left(x_{3}^{2} T\left(x_{2}\right)+2 x_{3} L\left(x_{2}\right)+x_{4}^{2} T\left(x_{2}\right)+2 x_{4} M\left(x_{2}\right)+2 N\left(x_{2}\right)\right) \partial_{1} f-2 \Lambda x_{3}^{2} \partial_{2} f \\
+4 \Lambda x_{1}+6 x_{4} A_{4}^{\prime}\left(x_{2}\right)+2 \Lambda \int N\left(x_{2}\right) d x_{2}+3 \int\left(M\left(x_{2}\right) A_{4}\left(x_{2}\right)\right) d x_{2}-6 C_{1}=0 \\
\Lambda x_{3}^{2} \partial_{1} f=0 \\
\Lambda x_{3}\left(x_{3} \partial_{3} f-1\right)=0 \\
\Lambda x_{3}^{2} \partial_{4} f-\Lambda x_{4}+3 A_{4}\left(x_{2}\right)=0
\end{array}\right.
$$

The second equation in (32) yields

$$
f=p\left(x_{2}, x_{3}, x_{4}\right)
$$

for some smooth function $p$. Replacing from the above Equation (33) into the third equation of (32) and integrating, we get

$$
p\left(x_{2}, x_{3}, x_{4}\right)=\ln \left(x_{3}\right)+q\left(x_{2}, x_{4}\right),
$$

where $q$ is a smooth function. We now replace the above expressions into the last equation of (32) and write it as a polynomial equation in the variable $x_{3}$, of the form

$$
\Lambda\left(\partial_{4} q\left(x_{2}, x_{4}\right)\right) x_{3}^{2}+\left(3 A_{4}\left(x_{2}\right)-\Lambda x_{4}\right)=0
$$

for all values of $x_{3}$. Therefore, $\partial_{4} q\left(x_{2}, x_{4}\right)=0$ and $3 A_{4}\left(x_{2}\right)-\Lambda x_{4}=0$, for all values of $x_{4}$, whence $\Lambda=0$, which can not occur. Therefore, we proved the following result, which completes the proof of Theorem.

Proposition 3. The expanding Ricci solitons on conformally flat Siklos metrics, as described in Theorem 2, are not gradient. 


\section{Siklos Metrics with Additional Killing Vector Fields}

We observe that the results of the present paper hold for a very large class of spacetimes. In fact, as showed by Equation (11), conformally flat Siklos metrics depend on four arbitrary smooth functions of one variable, namely, $T\left(x_{2}\right), L\left(x_{2}\right), M\left(x_{2}\right), N\left(x_{2}\right)$. We can measure the generality of these results by comparison with the corresponding study carried out in [11] for a one-parameter family of Siklos metrics, which were also homogeneous and, as such, admitted many additional symmetries.

The small price to pay for the generality of the actual results is that, as it could be expected, the explicit solutions we found, which exist for all conformally flat Siklos spacetimes, depend on the functions occurring in (11). Indeed, also Einstein examples within the class of Siklos metrics are not explicitly described, but identified through Equation (7).

All Siklos metrics admit at least one Killing vector field. In its pioneering paper [17] (p. 262), Siklos completely described, in terms of the defining function $H$, all metrics of the form (2) admitting one or more additional Killing vector fields. We shall report these special metrics below and give for each of them the explicit solutions of the Ricci soliton equation.

In each of the following cases we first write down the special form of the defining function $H$ for which additional Killing vector fields occur, following the notation we used in this paper for the global coordinates and the gravitational constant. We then write the conditions on functions $T, L, M, N$ occurring in Equation (11) $\left(L\left(x_{2}\right) \neq 0\right.$ in order to exclude the Einstein cases), ensuring that $H$ is of such required form; in other words, we completely determine the locally conformally flat examples within the special subclass we are considering.

Finally, we completely integrate (29) and (30) in these special cases, writing explicitly the forms of smooth functions $A_{1}$ and $A_{4}$ for the corresponding metrics. Such explicit forms, together with (31), completely describe the Ricci solitons on these conformally flat metrics. Following [17], $A\left(x_{i}\right)$ is an arbitrary smooth function of variables $x_{i}$, while the notation $A_{\alpha}\left(x_{i}\right)$ stands for a homogeneous function of degree $\alpha$ of the specified variables.

(1) $H=x_{2}^{-2} A\left(x_{3}, x_{4}\right)$.

By Proposition 2, such defining function $H$ determines a conformally flat metric if and only if $H$ is given by (11) with

$$
T\left(x_{2}\right)=T x_{2}^{-2}, \quad L\left(x_{2}\right)=L x_{2}^{-2}, \quad M\left(x_{2}\right)=M x_{2}^{-2}, \quad N\left(x_{2}\right)=N x_{2}^{-2},
$$

for some real constants $T, L(\neq 0), M, N$. Equations (29) and (30) can now be integrated. We treat separately the cases $T=0$ and $T>0$ (the case $T<0$ being similar to the latter).

For $T=0$ we find

$$
A_{4}\left(x_{2}\right)=-\frac{1}{6} \Lambda M \ln \left(x_{2}\right)+c_{1} x_{2}+c_{2},
$$

where, here and in the remaining part of the paper, $c_{i}$ denote some real constants. Substituting the above into (29) and integrating, we get

$$
A_{1}\left(x_{2}\right)=-\frac{\Lambda M^{2}}{12 x_{2}}\left(\ln \left(x_{2}\right)+1\right)-\frac{c_{1} M}{2} \ln \left(x_{2}\right)+\frac{1}{6 x_{2}}\left(3 c_{2} M+2 \Lambda N\right)+c_{3} .
$$

By integration of (29) and (30), the same argument for $T>0$ yields the explicit solutions

$$
A_{4}\left(x_{2}\right)=c_{1} x_{2}^{\frac{1-\sqrt{1+2 T}}{2}}+c_{2} x_{2}^{\frac{1+\sqrt{1+2 T}}{2}}-\frac{\Lambda M}{3 T}
$$

and

$$
\begin{aligned}
& A_{1}\left(x_{2}\right)=-\frac{1}{6 T x_{2}}\left\{3 c_{1} M(1-\sqrt{1+2 T}) x_{2}^{\frac{1-\sqrt{1+2 T}}{2}}+3 c_{2} M(1+\sqrt{1+2 T}) x_{2}^{\frac{1+\sqrt{1+2 T}}{2}}\right. \\
& \left.+\Lambda\left(M^{2}-2 T N\right)\right\}+c_{3} \text {. }
\end{aligned}
$$

(2) $H=A\left(x_{3}, x_{4}\right)$. 
It is easily seen that such defining function $H$ is of the form (11) if and only if

$$
T\left(x_{2}\right)=T, \quad L\left(x_{2}\right)=L, \quad M\left(x_{2}\right)=M, \quad N\left(x_{2}\right)=N,
$$

where $T, L(\neq 0), M, N$ are some real constants. We first integrate Equation (30) and then Equation (29).

If $T=0$ we get

$$
A_{4}\left(x_{2}\right)=\frac{1}{12} \Lambda M x_{2}^{2}+c_{1} x_{2}+c_{2}
$$

and

$$
A_{1}\left(x_{2}\right)=-\frac{\Lambda M^{2}}{72} x_{2}^{3}-\frac{c_{1} M}{4} x_{2}^{2}-\left(\frac{c_{2} M}{2}+\frac{\Lambda N}{3}\right) x_{2}+c_{3} .
$$

For $T>0$ we find

$$
A_{4}\left(x_{2}\right)=c_{1} e^{-\sqrt{\frac{T}{2}} x_{2}}+c_{2} e^{\sqrt{\frac{T}{2}} x_{2}}-\frac{\Lambda M}{3 T}
$$

and

$$
A_{1}\left(x_{2}\right)=\frac{\sqrt{2}}{6 \sqrt{T}}\left\{3 c_{1} M e^{-\sqrt{\frac{T}{2}} x_{2}}-3 c_{2} M e^{\sqrt{\frac{T}{2}} x_{2}}+\sqrt{2 T} \Lambda\left(M^{2}-2 T N\right) x_{2}\right\}+c_{3} .
$$

(3) $H=A_{2}\left(x_{2}, x_{3}, x_{4}\right)$.

Since $H$ must be a homogeneous function of degree 2 of variables $x_{2}, x_{3}, x_{4}$, Equation (11) holds if and only if

$$
T\left(x_{2}\right)=T, \quad L\left(x_{2}\right)=L x_{2}, \quad M\left(x_{2}\right)=M x_{2}, \quad N\left(x_{2}\right)=N x_{2}^{2}
$$

for some real constants $T, L(\neq 0), M, N$.

For $T=0$ we find

$$
A_{4}\left(x_{2}\right)=\frac{1}{36} \Lambda M x_{2}^{3}+c_{1} x_{2}+c_{2}
$$

and

$$
A_{1}\left(x_{2}\right)=-\frac{\Lambda M^{2}}{360} x_{2}^{5}-\left(6 M c_{1}+9 N \Lambda\right) x_{2}^{3}-\frac{1}{4} c_{2} M x_{2}^{2}+c_{3},
$$

while for $T>0$ by integration we get

$$
A_{4}\left(x_{2}\right)=c_{1} e^{-\sqrt{\frac{T}{2}} x_{2}}+c_{2} e^{\sqrt{\frac{T}{2}} x_{2}}-\frac{\Lambda M}{3 T} x_{2}
$$

and

$$
A_{1}\left(x_{2}\right)=\frac{c_{1} M}{T}\left(\sqrt{\frac{T}{2}} x_{2}+1\right) e^{-\sqrt{\frac{T}{2}} x_{2}}-\frac{c_{2} M}{T}\left(\sqrt{\frac{T}{2}} x_{2}-1\right) e^{\sqrt{\frac{T}{2}} x_{2}}+\frac{\Lambda}{18 T}\left(M^{2}-2 T N\right) x_{2}^{3} .
$$

(4) $H=A\left(x_{2}, x_{3}\right)$.

The function $H$ of a conformally flat Siklos metric is of the above form if and only if (11) holds with $T\left(x_{2}\right)=M\left(x_{2}\right)=0$, for arbitrary smooth functions $L\left(x_{2}\right)(\neq 0)$ and $N\left(x_{2}\right)$. Then, integrating (30) we obtain

$$
A_{4}\left(x_{2}\right)=c_{1} x_{2}+c_{2}
$$

which, replaced into (29) yields

$$
A_{1}\left(x_{2}\right)=-\frac{\Lambda}{3} \int N\left(x_{2}\right) d x_{2}+c_{3}
$$

(5) $H=A_{\alpha}\left(x_{3}, x_{4}\right), \alpha \neq-2$.

If $H$, as described by (11), is a homogeneous function of order $\alpha$ of variables $x_{3}, x_{4}$, then one of the following cases must occur:

(a) $\quad \alpha=2, T\left(x_{2}\right)=T$ and $L\left(x_{2}\right)=M\left(x_{2}\right)=N\left(x_{2}\right)=0$. But then, as $L\left(x_{2}\right)=0$, the metric is Einstein. 
(b) $\quad \alpha=1, T\left(x_{2}\right)=N\left(x_{2}\right)=0$ and $L\left(x_{2}\right)=L, M\left(x_{2}\right)=M$, for some real constants $L(\neq 0), M$.

In the latter case, $A_{4}\left(x_{2}\right)$ is of the form (34) and $A_{1}\left(x_{2}\right)=c_{3}$.

(6) $H=A\left(x_{3}\right) e^{x_{4}}$.

This form of $H$ is not compatible with (11).

(7) $H=A_{-2}\left(x_{3}, x_{4}\right)$.

This form of $H$ is not compatible with (11).

(8) $H=A\left(x_{3}\right)$.

Clearly, this may be treated as a special case of the above case 4). explicitly, we get that $A_{4}\left(x_{2}\right)$ is described by (34) and $A_{1}\left(x_{2}\right)=c_{3}$.

(9) $H=A\left(x_{2}\right) x_{3}^{2}$.

This form of $H$ is not compatible with (11) (as the coefficients of $x_{3}^{2}$ and $x_{4}^{2}$ in (11) must coincide).

(10) $H=x_{2}^{2 \beta-2} A\left(x_{3} x_{2}^{\beta}\right)$.

In order of the above $H$ to be compatible with the form (11), we must have

$$
T\left(x_{2}\right)=M\left(x_{2}\right)=0, \quad L\left(x_{2}\right)=x_{2}^{2 \beta+2} L 1\left(x_{2}\right), \quad N\left(x_{2}\right)=x_{2}^{2 \beta+2} N 1\left(x_{2}\right),
$$

for some smooth functions $L 1, N 1$. However, being such functions arbitrary, this means that functions $L\left(x_{2}\right), N\left(x_{2}\right)$ are arbirtrary. Hence, we get the same explicit solutions of the above case 4$)$.

(11) $H= \pm x_{3}^{\alpha}$.

This is the homogeneous case already treated in [11] for arbitrary values of the parameter $\alpha$. In the notation of this paper, we have that $H= \pm x_{3}^{\alpha}$ corresponds to a conformally flat Siklos metric if and only if $\alpha=1$ and (11) holds for $T\left(x_{2}\right)=M\left(x_{2}\right)=N\left(x_{2}\right)=0$ and $L\left(x_{2}\right)= \pm 1$. Then, $A_{4}\left(x_{2}\right)$ is given by (34) and $A_{1}\left(x_{2}\right)=c_{3}$.

(12) $H= \pm x_{3}^{-2}$.

This form of $H$ is not compatible with (11).

\section{Conclusions}

The study of Ricci solitons in Lorentzian settings is an active field of research in Differential Geometry and Theoretical Physics. On the one hand, this investigation led to determine some strong restrictions on the geometry of a Lorentzian manifold satisfying the Ricci soliton equation when the Ricci soliton is of gradient type. This fact is particularly evident in the case of locally conformally flat Lorentzian manifolds. On the other hand, such rigidity results make natural to ask whether they extend to the case where the Ricci soliton is not gradient.

The present paper gives a contribution in this line of work, providing a large class of locally conformally flat Lorentzian examples, all of which are Ricci solitons, but whose geometry is not strongly restricted by the Ricci soliton equation. Consequently, they do not fit into the classification obtained in [6] for Lorentzian gradient Ricci solitons.

Funding: Author partially supported by funds of the University of Salento and INDAM (GNSAGA).

Conflicts of Interest: The author declares no conflict of interest. 


\section{References}

1. Cao, H.-D. Recent progress on Ricci solitons. Adv. Lect. Math. 2009, 11, 1-38.

2. Hamilton, R.S. The Ricci flow on surfaces. Contemp. Math. 1988, 71, 237-261.

3. Akbar, M.M.; Woolgar, E. Ricci solitons and Einstein-scalar field theory. Class. Quantum. Grav. 2009, 26, 055015. [CrossRef]

4. Batat, W.; Brozos-Vazquez, M.; Garcia-Rio, E.; Gavino-Fernandez, S. Ricci solitons on Lorentzian manifolds with large isometry groups. Bull. London Math. Soc. 2011, 43, 1219-1227. [CrossRef]

5. Brozos-Vazquez, M.; Calvaruso, G.; Garcia-Rio, E.; Gavino-Fernandez, S. Three-dimensional Lorentzian homogeneous Ricci solitons. Israel J. Math. 2012, 188, 385-403. [CrossRef]

6. Brozos-Vazquez, M.; Garcia-Rio, E.; Gavino-Fernandez, S. Locally conformally flat Lorentzian gradient Ricci solitons. J. Geom. Anal. 2013, 23, 1196-1212. [CrossRef]

7. Brozos-Vazquez, M.; Garcia-Rio, E.; Gavino-Fernandez, S.; Gilkey, P. The structure of the Ricci tensor on locally homogeneous Lorentzian gradient Ricci solitons. Proc. Roy. Soc. Edinb. Sect. 2018, 148, 461-482. [CrossRef]

8. Calvaruso, G. Oscillator spacetimes are Ricci solitons. Nonlinear Anal. 2016, 140, 254-269. [CrossRef]

9. Calvaruso, G. The Ricci soliton equation and the structure of homogeneous Gödel-type spacetimes. J. Math. Anal. Appl. 2018, 465, 1112-1133. [CrossRef]

10. Calvaruso, G. On semi-direct extensions of the Heisenberg group. Collect. Math. 2020, in press. [CrossRef]

11. Calvaruso, G. Siklos spacetimes as homogeneous Ricci solitons. Class. Quantum Grav. 2019, 36, 095011. [CrossRef]

12. Calvaruso, G.; Fino, A. Ricci solitons and geometry of four-dimensional non-reductive homogeneous spaces. Canad. J. Math. 2012, 64, 778-804. [CrossRef]

13. Calvaruso, G.; Fino, A. Four-dimensional pseudo-Riemannian homogeneous Ricci solitons. Int. J. Geom. Methods Mod. Phys. 2015, 12, 1550056. [CrossRef]

14. Calvaruso, G.; Zaeim, A. A complete classification of Ricci and Yamabe solitons of non-reductive homogeneous 4-spaces. J. Geom. Phys. 2014, 80, 15-25. [CrossRef]

15. Case, J.S. Singularity theorems and the Lorentzian splitting theorem for the Bakry-Emery-Ricci tensor. J. Geom. Phys. 2010, 60, 477-490. [CrossRef]

16. Pina, R.; Tenenblat, K. On solutions of the Ricci curvature and the Einstein equation. Israel J. Math. 2009, 171, 61-76. [CrossRef]

17. Siklos, S.T.C. Lobatchevski plane gravitational waves. In Galaxies, Axisymmetric Systems and Relativity; MacCallum, M.A.H., Ed.; Cambridge University Press: Cambridge, UK, 1985.

18. Podolski, J. Interpretation of the Siklos solutions as exact gravitational waves in the anti-de Sitter universe. Class. Quantum Grav. 1998, 15, 719-733. [CrossRef]

19. Ozsváth, I.; Robinson, I.; Rózga, K. Plane-fronted gravitational and electromagnetic waves in spaces with cosmological constant. J. Math. Phys. 1985, 26, 1755-1761. [CrossRef]

(C) 2020 by the authors. Licensee MDPI, Basel, Switzerland. This article is an open access article distributed under the terms and conditions of the Creative Commons Attribution (CC BY) license (http://creativecommons.org/licenses/by/4.0/). 